PROCEEDINGS OF THE

AMERICAN MATHEMATICAL SOCIETY

Volume 138, Number 5, May 2010, Pages 1861-1876

S 0002-9939(09)10238-1

Article electronically published on December 21, 2009

\title{
THE LOOP GROUP AND THE COBAR CONSTRUCTION
}

\author{
KATHRYN HESS AND ANDREW TONKS
}

(Communicated by Brooke Shipley)

\begin{abstract}
We prove that for any 1-reduced simplicial set $X$, Adams' cobar construction $\Omega C X$ on the normalised chain complex of $X$ is naturally a strong deformation retract of the normalised chains $C G X$ on the Kan loop group $G X$. In order to prove this result, we extend the definition of the cobar construction and actually obtain the existence of such a strong deformation retract for all 0 -reduced simplicial sets.
\end{abstract}

\section{INTRODUCTION}

There are two classical differential graded algebra models for the loop space on a 1-reduced simplicial set $X$ : Adams' cobar construction $\Omega C X$ on the normalised chain complex [1, and the normalised chains $C G X$ on the Kan loop group $G X$ [7]. Both of these models are (weakly) equivalent to $C \Omega|X|$, the chains on the loop space of the realisation $|X|$.

In this article we show that $\Omega C X$ is actually a strong deformation retract of $C G X$, opening up the possibility of applying the tools of homological algebra to transferring perturbations of algebraic structure from the latter to the former.

Theorem. For any 1 -reduced simplicial set $X$ there is a natural strong deformation retract of chain complexes

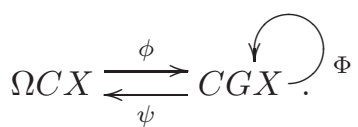

Here $\psi \phi$ is the identity map on $\Omega C X$ and $\Phi$ is a chain homotopy from $\phi \psi$ to the identity map on CGX. Furthermore both $\phi$ and $\psi$ are homomorphisms of differential graded algebras.

In particular, $\Omega C X$ is isomorphic to a subdifferential graded algebra of $C G X$, and both $\phi$ and $\psi$ induce isomorphisms of algebras in homology.

Remark 1. Let $X$ and $Y$ be 1-reduced simplicial sets, and let $f: G X \rightarrow G Y$ be a simplicial map (not necessarily a homomorphism). The theorem above gives us a natural way to construct a chain-level model of $f$. Indeed, if we set

$$
\xi=\psi \circ C f \circ \phi: \Omega C X \rightarrow \Omega C Y,
$$

Received by the editors March 13, 2009.

2010 Mathematics Subject Classification. Primary 55P35; Secondary 16T05, 18G30, 55U10, 57T05, 57T30.

Key words and phrases. Loop space, cobar construction, strong deformation retract, acyclic models.

(C)2009 American Mathematical Society
Reverts to public domain 28 years from publication 1861 
then

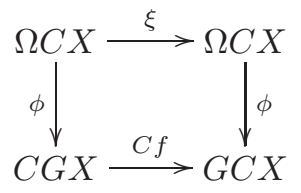

commutes up to natural chain homotopy.

Remark 2. It was proved in 6 that if $X$ is a simplicial suspension, then $\Omega C X$ is naturally a primitively generated Hopf algebra, and the chain algebra map $\phi$ : $\Omega C X \rightarrow C G X$ also respects comultiplicative structure. In this case the strong deformation retract of the theorem above is actually Eilenberg-Zilber data [3, which implies that the chain algebra map $\psi: C G X \rightarrow \Omega C X$ is also strongly homotopy comultiplicative [ibid.].

In order to prove the theorem above, we extend the definition of the cobar construction and actually obtain the existence of such a strong deformation retract for all 0-reduced simplicial sets.

The homomorphism $\phi$, which we recall in the first section of this article, was first described by Szczarba [11 in the language of twisting cochains. Given a simplicial set $X$ that is 0-reduced but not necessarily 1-reduced, he gives an explicit, though somewhat complicated, formula for a twisting cochain,

$$
\alpha_{\phi}: C X \rightarrow C G X
$$

that is based on the universal twisting function $\tau: X \rightarrow G X$ and that gives rise in the usual way to an algebra homomorphism

$$
\phi: \Omega C X \rightarrow C G X .
$$

In degree zero the cobar construction is a free associative algebra on symbols given by the nondegenerate 1-simplices of $X$, while the right-hand side is the group ring on the free group on the same symbols. In the first section of the paper we observe that if $X$ is not 1-reduced, then we may perform a change of rings along $\phi_{0}$, obtaining an extended cobar construction $\hat{\Omega} C X$, together with an algebra homomorphism

$$
\phi: \hat{\Omega} C X \rightarrow C G X
$$

that is an isomorphism in degree zero.

In the second section we introduce the retraction map $\psi$ from the chains on the loop group to the extended cobar construction, for which we provide an explicit recursive formula. We prove in fact that $\psi$ is a natural homomorphism of chain algebras and a one-sided inverse of the Szczarba map $\phi$. It is surprising that such a map has not been previously observed in the literature.

In the third section we complete the strong deformation retraction (11) by defining the natural homotopy $\Phi$. For this, we use the acyclic models for the loop group on 0-reduced simplices studied by Morace and Prouté [10.

\section{Preliminaries}

1.1. Simplicial notions and notation. A simplicial set $X$ is a contravariant functor from the category of finite nonempty ordinals $\Delta$ to the category of sets; 
more prosaically it is a sequence of sets $X_{n}, n \geq 0$, and specified face and degeneracy operators

$$
d_{i}: X_{n} \rightarrow X_{n-1}, \quad s_{i}: X_{n} \rightarrow X_{n+1} \quad(0 \leq i \leq n)
$$

satisfying the simplicial identities; see for example 9 . A simplicial set is $n$-reduced if $X_{k} \cong\{*\}$ for $k \leq n$. The notions of simplicial group and simplicial objects in other categories are analogous.

Given an element $x \in X_{n}$ and any composite $\theta$ of simplicial face and degeneracy operators, represented by a monotonic function $f:\{0, \ldots, m\} \rightarrow\{0, \ldots, n\}$ in $\Delta$, we also write

$$
\theta(x)=x_{(f(0), \ldots, f(m))}
$$

for the corresponding element of $X_{m}$. We may write the face and degeneracy maps themselves, for example, as

$$
\begin{aligned}
d_{i}(x) & =x_{\left(0, \ldots, \hat{i}_{, \ldots, n)},\right.} \\
s_{i}(x) & =x_{(0, \ldots, i, i, \ldots, n)} .
\end{aligned}
$$

The simplicial relations imply that any simplicial operator $X_{n} \rightarrow X_{m}$ has normal form

$$
\theta=s_{i_{1}} \ldots s_{i_{q}} d_{j_{1}} \ldots d_{j_{r}}
$$

with $i_{k}>i_{k+1}$ and $j_{k}<j_{k+1}$ for all $k$. In this form, the corresponding derived operator $X_{n+1} \rightarrow X_{m+1}$ is

$$
\theta^{\prime}=s_{i_{1}+1} \ldots s_{i_{q}+1} d_{j_{1}+1} \ldots d_{j_{r}+1} .
$$

An operator $\theta$ is frontal if it contains no $d_{0}$; such operators satisfy $\theta^{\prime} s_{0}=s_{0} \theta$.

1.2. The cobar construction. We introduce a slightly extended definition of the cobar construction, which will be better suited for applying to the normalised chain complex on a 0-reduced simplicial set. Our definition generalises the classical construction of Adams, with which it agrees for simply connected coalgebras.

Let $(C, \partial, \Delta)$ be a connected differential graded coalgebra over a commutative $\operatorname{ring} R$, so that $C_{0}=R$. We suppose furthermore that $C$ is $R$-free in each degree. Consider the ring $B$ given by the free associative $R$-algebra on the desuspension of $C_{1}$

$$
B=T\left(s^{-1} C_{1}\right)=\bigoplus_{k \geq 0}\left(s^{-1} C_{1}\right)^{\otimes k} .
$$

Fix an $R$-basis $\left\{x_{j} \mid j \in J\right\}$ of $C_{1}$, and let $A$ be the ring obtained from $B$ by freely adjoining inverses $\xi_{j}$ of all elements of the form $1+s^{-1} x_{j}$, for all $j \in J$. Explicitly,

$$
A=T_{B}\left(\xi_{j} \mid j \in J\right) /\left(\xi_{j} \otimes\left(1+s^{-1} x_{j}\right)=1=\left(1+s^{-1} x_{j}\right) \otimes \xi_{j}\right) .
$$

Observe that the relations may also be expressed in the form

$$
\xi_{j} \otimes s^{-1} x_{j}=1-\xi_{j}=s^{-1} x_{j} \otimes \xi_{j} .
$$

The ring $A$ may be regarded as a differential graded algebra concentrated in degree zero. The graded algebra underlying the extended cobar construction $\left(\hat{\Omega} C, \partial^{\Omega}\right)$ is then

$$
\begin{aligned}
\hat{\Omega} C & =T_{A}\left(s^{-1} C_{\geq 2}\right) \\
& =\bigoplus_{k \geq 0} A \otimes\left(s^{-1} C_{\geq 2} \otimes A\right)^{\otimes k} .
\end{aligned}
$$


Each $R$-module $(\hat{\Omega} C)_{n}$ is generated by words

$$
a=a_{1} \otimes \cdots \otimes a_{r}, \quad\left|a_{i}\right|=n_{i}, \quad|a|=n=\sum n_{i},
$$

where either $a_{i}=s^{-1} c$ for some $R$-basis element $c \in C_{n_{i}+1}$ or $n_{i}=0$ and $a_{i}=\xi_{j}$ for some $j \in J$. This $R$-module is free on those words in which $\xi_{j}$ does not appear adjacent to $s^{-1} x_{j}$. The algebra multiplication is induced by a concatenation of words, extended bilinearly to $\hat{\Omega} C$, modulo the relation that $\xi_{j}$ is inverse to $1+s^{-1} x_{j}$.

The differential $\partial^{\Omega}$ on $\hat{\Omega} C$ is the derivation of $A$-algebras that is specified by

$$
\partial_{n}^{\Omega} s^{-1} c=-s^{-1} d c+(-1)^{\left|c_{i}\right|} s^{-1} c_{i} \otimes s^{-1} c^{i}
$$

for all basis elements $c \in C_{n+1}$ and all $n \geq 1$, where $\Delta(c)=1 \otimes c+c \otimes 1+c_{i} \otimes c^{i}$ (using the Einstein summation convention). Note that $\partial^{\Omega}$ is necessarily zero on elements of $A$.

The unit $1 \in(\hat{\Omega} C)_{0}$ is identified with the empty word, via the isomorphism $R \cong\left(s^{-1} C_{1}\right)^{\otimes 0}$.

Remark 3. If $C$ is simply connected so that $C_{1}=\{0\}$ and therefore $A=B=R$, then $\hat{\Omega} C$ coincides with the usual cobar construction $\Omega C$ defined by Adams.

1.3. The Kan loop group. Let $X$ be a 0 -reduced simplicial set and $G$ a simplicial group. A twisting function $\tau: X \rightarrow G$ is a collection of functions of degree -1 ,

$$
\left\{\tau: X_{n+1} \rightarrow G_{n} \mid n \geq 0\right\}
$$

satisfying

$$
\begin{aligned}
\tau s_{0} x & =1, \\
s_{i} \tau x & =\tau s_{i+1} x, \\
d_{0} \tau x & =\tau d_{0} x^{-1} \cdot \tau d_{1} x, \\
d_{i} \tau x & =\tau d_{i+1} x \quad \text { if } i \geq 1 .
\end{aligned}
$$

Let $G X$ denote the Kan loop group on $X$, which is a simplicial group that models the space of based loops on the geometric realization of $X$ (see [7, 9]). There is a universal twisting function

$$
\begin{aligned}
\tau: X_{n+1} & \rightarrow(G X)_{n}=\mathbf{F}\left(X_{n+1}\right) / \mathbf{F}\left(s_{0} X_{n}\right), \\
\tau x & =[x],
\end{aligned}
$$

sending $x \in X_{n+1}$ to the class of the corresponding generator in the quotient of free groups, and the simplicial structure on $G X$ is defined by (2)-(4).

Recall that the normalised chain complex $C G$ on a simplicial group $G$ also has a differential graded algebra structure, with multiplication given by the shuffle map and the multiplication in $G$,

$$
m: C G \otimes C G \longrightarrow C(G \times G) \longrightarrow C G
$$

that is,

$$
m(g \otimes h)=\sum(-1)^{\operatorname{sgn}(\mu, \nu)} s_{\mu(q)} \ldots s_{\mu(1)} g \cdot s_{\nu(p)} \ldots s_{\nu(1)} h, \quad g \in G_{p}, h \in G_{q}
$$

where the summation is over all $(p, q)$-shuffles $(\mu, \nu) \in \operatorname{Shuff}(p, q)$.

The following proposition is the motivation for our extension of the cobar construction. Recall that for any simplicial set $X$, the degree $n$ part of its normalised 
chain complex, $C_{n} X$, is the free abelian group on the set of all nondegenerate $n$ simplices of $X$ and that $C X$ has a comultiplication $\Delta: C X \rightarrow C X \otimes C X$ given by the Alexander-Whitney diagonal approximation

$$
\Delta(x)=x_{(0)} \otimes x+\sum_{i=1}^{n-1} x_{(0, \ldots, i)} \otimes x_{(i, \ldots, n)}+x \otimes x_{(n)}
$$

for $x \in X_{n}, n \geq 1$, and with $\Delta(x)=x \otimes x$ for $x \in X_{0}$. In particular, if $X$ is 0-reduced, then $C X$ is a connected, differential graded coalgebra over $\mathbb{Z}$.

Proposition 4. Let $X$ be a 0-reduced simplicial set and $G X$ its Kan loop group. Then there is an isomorphism of rings

$$
(\hat{\Omega} C X)_{0} \underset{\psi_{0}}{\stackrel{\phi_{0}}{\rightleftarrows}}(C G X)_{0}
$$

determined by

$$
\begin{aligned}
\psi_{0}(\tau x) & =\xi_{x}, & \psi_{0}\left(\tau x^{-1}\right) & =1+s^{-1} x, \\
\phi_{0}\left(s^{-1} x\right) & =\tau x^{-1}-1, & \phi_{0}\left(\xi_{x}\right) & =\tau x .
\end{aligned}
$$

Proof. The proof is straightforward. Note that if $x$ is the degenerate element $s_{0}(*)$, then the four equations yield $\psi_{0}(1)=1, \phi_{0}(0)=0, \phi_{0}(1)=1$. In degree 0 the multiplication (5) is just $m(g \otimes h)=g \cdot h$, and we have

$$
\begin{aligned}
\psi_{0}\left(\tau x_{1}^{\alpha_{1}} \ldots \tau x_{r}^{\alpha_{r}}\right) & =\psi_{0}\left(\tau x_{1}^{\alpha_{1}}\right) \otimes \cdots \otimes \psi_{0}\left(\tau x_{r}^{\alpha_{r}}\right), \\
\phi_{0}\left(a_{1} \otimes \cdots \otimes a_{r}\right) & =\phi_{0}\left(a_{1}\right) \cdots \phi_{0}\left(a_{r}\right),
\end{aligned}
$$

where $x_{i} \in X_{1}, \alpha_{i}= \pm 1$ and $a_{i}=s^{-1} x_{i}$ or $\xi_{x_{i}}$. This is well defined: $\psi_{0}(g)$ is inverse to $\psi_{0}\left(g^{-1}\right)$ for all $g \in(G X)_{0}$ and $\phi_{0}\left(\xi_{x}\right)$ is inverse to $\phi_{0}\left(1+s^{-1} x\right)$ for all $x \in X_{1} \backslash\left\{s_{0}(*)\right\}$. It is also clear that the composites $\phi_{0} \psi_{0}$ and $\psi_{0} \phi_{0}$ are the respective identity maps:

$$
\begin{aligned}
\phi_{0} \psi_{0}(\tau x) & =\phi_{0}\left(\xi_{x}\right)=\tau x, & \phi_{0} \psi_{0}\left(\tau x^{-1}\right) & =\phi_{0}\left(1+s^{-1} x\right)=\tau x^{-1}, \\
\psi_{0} \phi_{0}\left(s^{-1} x\right) & =\psi_{0}\left(\tau x^{-1}-1\right)=s^{-1} x, & \psi_{0} \phi_{0}\left(\xi_{x}\right) & =\psi_{0}(\tau x)=\xi_{x} .
\end{aligned}
$$

1.4. The Szczarba map. A map of differential graded algebras

$$
\phi: \Omega C X \rightarrow C G X
$$

was given explicitly by Szczarba in the language of twisting cochains. The following definition, lemma and theorem are from sections 2 and 3 of Szczarba's paper [11, adapted slightly to define a map on the extended cobar construction

$$
\phi: \hat{\Omega} C X \rightarrow C G X
$$

that extends the isomorphism $\phi_{0}$ of Proposition 4

Let $S_{n}$ be the set of $n$ ! sequences of integers

$$
i=\left(i_{1}, \ldots, i_{n}\right) \quad \text { such that } 0 \leq i_{k} \leq n-k \text { for each } k .
$$

In particular, $i_{n}=0$. The sign of such a sequence $i \in S_{n}$ is

$$
(-1)^{\sum i}, \quad \text { where } \quad \sum i=i_{1}+i_{2}+\cdots+i_{n} .
$$


Definition 5. Given a twisting function $\tau: X \rightarrow G$, the Szczarba operators are the functions

$$
\mathrm{Sz}_{i}: X_{n+1} \longrightarrow G_{n}, \quad i=\left(i_{1}, \ldots, i_{n}\right) \in S_{n},
$$

given by the following product in $G_{n}$ :

$$
\mathrm{Sz}_{i} x=D_{0 ; i}^{n+1} \tau x^{-1} \cdot D_{1 ; i}^{n+1} \tau d_{0} x^{-1} \cdots D_{n ; i}^{n+1} \tau d_{0}^{n} x^{-1} .
$$

Here the operators $D_{j ; i}^{n+1}: G_{n-j} \rightarrow G_{n}$ for $i \in S_{n}, j=0, \ldots, n$, are defined as

$$
\begin{aligned}
D_{0 ;()}^{1} & =I d_{G_{0}}, \\
D_{j ; i_{1}, \ldots, i_{n}}^{n+1} & = \begin{cases}D_{j ; i_{2}, \ldots, i_{n}}^{n} s_{0} d_{i_{1}-j} & \text { if } j<i_{1}, \\
D_{j ; i_{2}, \ldots, i_{n}}^{n !} & \text { if } j=i_{1}, \\
D_{j-1 ; i_{2}, \ldots, i_{n}}^{n \prime} s_{0} & \text { if } j>i_{1} .\end{cases}
\end{aligned}
$$

As simplicial operators these are all frontal: defining $D_{j ; i}^{n+1}: X_{n-j} \rightarrow X_{n}$ in the same way, one has $D_{j ; i}^{n+1} \tau=\tau D_{j ; i}^{n+1^{\prime}}: X_{n-j+1} \rightarrow G_{n}$.

Lemma 6. The Szczarba operators satisfy

$$
\begin{array}{llc}
d_{0} S z_{i_{1}, \ldots, i_{n}} & = & S z_{i_{2}, \ldots, i_{n}} d_{i_{1}+1}, \\
d_{k} S z_{i_{1}, \ldots, i_{k}, i_{k+1}, \ldots, i_{n}} & = & d_{k} S z_{i_{1}, \ldots, i_{k+1}, i_{k}-1, \ldots, i_{n}} \quad \text { if } i_{k}>i_{k+1}, \\
d_{n} S z_{i_{1}, \ldots, i_{n}} x & = & s_{\mu} S z_{i^{\prime}} x_{(0, \ldots, r)} \cdot s_{\nu} S z_{i^{\prime \prime}} x_{(r, \ldots, n+1)} .
\end{array}
$$

In the last equation the sequences $i^{\prime}, i^{\prime \prime}$, the integer $r$ and the $(r-1, n-r)$-shuffle $(\mu, \nu)$ are defined by a certain bijection

$$
\begin{array}{ccc}
S_{n} & \cong & \bigcup_{r=1}^{n} \operatorname{Shuff}(r-1, n-r) \times S_{r-1} \times S_{n-r}, \\
i & \mapsto & \left((\mu, \nu), i^{\prime}, i^{\prime \prime}\right)
\end{array}
$$

(see [11, Lemma 3.3]), which respects parity as follows:

$$
n+\sum i=r+\operatorname{sgn}(\mu, \nu)+\sum i^{\prime}+\sum i^{\prime \prime}(\bmod 2) .
$$

Note that Szczarba's sign conventions differ slightly from ours and that his inductively-defined parity $\epsilon(i, n+1)$ is in fact just $n+\sum i$.

Theorem 7. For any twisting function $\tau: X \rightarrow G$ on a 0 -reduced simplicial set $X$, there is a canonical homomorphism of differential graded algebras defined by

$$
\begin{aligned}
\phi: \hat{\Omega} C X & \longrightarrow C G, \\
\phi_{0}\left(\xi_{x_{1}}\right) & =\tau x_{1}, \\
\phi_{0}\left(s^{-1} x_{1}\right) & =\tau\left(x_{1}\right)^{-1}-1, \\
\phi_{n}\left(s^{-1} x_{n+1}\right) & =\sum_{i \in S_{n}}(-1)^{\sum i} S z_{i} x \quad(n \geq 1),
\end{aligned}
$$

for $x_{n+1} \in X_{n+1}$.

Proof. The map $\phi$ extends linearly and multiplicatively via

$$
\phi_{p+q}(a \otimes b)=m\left(\phi_{p}(a) \otimes \phi_{q}(b)\right), \quad|a|=p,|b|=q,
$$


where $m$ is the multiplication (15), to all of $\hat{\Omega} C X$. We show $\phi$ is a chain map, i.e., that $\partial_{n} \phi_{n}=\phi_{n-1} \partial_{n}^{\Omega}$. For $x \in X_{2}$ we can write

$$
\begin{aligned}
\partial_{1}^{\Omega} s^{-1} x & =-s^{-1} d_{0} x+s^{-1} d_{1} x-s^{-1} d_{2} x-s^{-1} x_{(0,1)} \otimes s^{-1} x_{(1,2)} \\
& =\left(1+s^{-1} d_{1} x\right)-\left(1+s^{-1} x_{(0,1)}\right) \otimes\left(1+s^{-1} x_{(1,2)}\right)
\end{aligned}
$$

and so we have, by Lemma 6 ,

$$
\begin{aligned}
\partial_{1} \phi_{1} s^{-1} x & =\partial_{1} \mathrm{Sz}_{0} x=d_{0} \mathrm{Sz}_{0} x-d_{1} \mathrm{Sz}_{0} x=\mathrm{Sz}_{()} d_{1} x-\mathrm{Sz}_{()} x(0,1) \\
& =\tau\left(d_{1} x\right)^{-1}-\tau\left(x_{(0,1)}\right)^{-1} \cdot \tau\left(x_{(1,2)}\right)^{-1}=x_{(1,2)} \partial_{1}^{\Omega} s^{-1} x .
\end{aligned}
$$

For $x \in X_{n+1}$ the argument is essentially the same. We have

$$
\begin{aligned}
\partial_{n} \phi_{n} s^{-1} x & =\sum_{i \in S_{n}}(-1)^{\sum i} \partial_{n} \mathrm{Sz}_{i} x \\
& =\sum_{i \in S_{n}}(-1)^{\sum i}\left(\sum_{k=0}^{n}(-1)^{k} d_{k} \mathrm{Sz}_{i} x\right),
\end{aligned}
$$

where, by Lemma 6. all the terms for $0<k<n$ cancel, and the terms for $k=0, n$ may be rewritten as

$$
\begin{aligned}
& \sum_{\substack{0 \leq i_{1} \leq n-1 \\
i \in S_{n-1}}}(-1)^{i_{1}+\sum i} \mathrm{Sz}_{i} d_{i_{1}+1} x+\sum_{\substack{1 \leq r \leq n \\
(\mu, \nu), i^{\prime}, i^{\prime \prime}}}(-1)^{n+\sum i} s_{\mu} \mathrm{Sz}_{i^{\prime}} x_{(0, \ldots, r)} \cdot s_{\nu} \mathrm{Sz}_{i^{\prime \prime}} x_{(r, \ldots, n+1)} \\
&= \sum_{1 \leq r \leq n}(-1)^{r-1} \phi_{n-1} s^{-1} d_{r} x+\sum_{\substack{1 \leq r \leq n\\
}}(-1)^{r} m\left(\phi_{r-1} s^{-1} x_{(0, \ldots, r)} \otimes \phi_{n-r} s^{-1} x_{(r, \ldots, n+1)}\right) \\
& \quad-1 \otimes \phi_{n-1} s^{-1} x_{(1, \ldots, n+1)}+(-1)^{n} \phi_{n-1} s^{-1} x_{(0, \ldots, n)} \otimes 1 \\
&= \phi_{n-1}\left(\sum_{r=0}^{n+1}(-1)^{r-1} s^{-1} d_{r} x+\sum_{r=1}^{n}(-1)^{r} s^{-1} x_{(0, \ldots, r)} \otimes s^{-1} x_{(r, \ldots, n+1)}\right) \\
&= \phi_{n-1} \partial_{n}^{\Omega} x .
\end{aligned}
$$

We will need one further property of the Szczarba operators.

Lemma 8. For all $x \in X_{n+1}$ and $i \in S_{n}$, the following product in $G_{n}$ is degenerate:

$$
D_{0 ; i}^{n+1} \tau x \cdot S z_{i} x=D_{1 ; i}^{n+1} \tau d_{0} x^{-1} \cdots D_{n ; i}^{n+1} \tau d_{0}^{n} x^{-1} .
$$

Proof. For any sequence $i \in S_{n}$ we will show by induction that $D_{j ; i}^{n+1}$ is $s_{\kappa(i)-1^{-}}$ degenerate for all $j>0$, where $\kappa(i)$ is the least integer such that $i_{\kappa(i)}=0$. If $i_{1}=0$, so that $\kappa(i)=1$, then by (6),

$$
D_{j ; i}^{n+1}=D_{j-1 ; i_{2}, \ldots, i_{n}}^{n \prime} s_{0}=s_{0} D_{j-1 ; i_{2}, \ldots, i_{n}}^{n}
$$

for all $j>i_{1}=0$. If $i_{1}>0$, so that $\kappa(i)>1$, then $\kappa\left(i_{2}, \ldots, i_{n}\right)=\kappa(i)-1$ and we know that $D_{j ; i_{2}, \ldots, i_{n}}^{n}$ (if $j>0$ ) and $D_{j-1 ; i_{2}, \ldots, i_{n}}^{n}$ (if $j>1$ ) are $s_{\kappa(i)-2}$-degenerate by the inductive hypothesis. The corresponding derived operators are therefore $s_{\kappa(i)-1}$-degenerate and, by (6), so is $D_{j ; i}^{n+1}$ for all $j>0$. 


\section{THE RETRACTION MAP}

2.1. Definition of the map. Let $X$ be a 0 -reduced simplicial set. We introduce in this section a map of differential graded algebras

$$
\psi: C G X \longrightarrow \hat{\Omega} C X
$$

between the chains on the loop group and the extended cobar construction, which

is a retraction of the Szczarba map $\phi$. The map $\psi$ is uniquely determined by the relation

$$
\psi_{n}(\tau x \cdot g)=\psi_{n}(g)-\sum_{i=0}^{n} s^{-1} x_{(0, \ldots, i+1)} \otimes \psi_{n-i}\left(\tau d_{1}^{i} x \cdot d_{0}^{i} g\right)
$$

for $x \in X_{n+1}$ and $g \in(G X)_{n}$. Note that the $i=0$ term on the right-hand side is $s^{-1} x_{(0,1)} \otimes \psi_{n}(\tau x \cdot g)$. In fact $\psi$ may be expressed inductively, on the degree $n$ and the word length in $(G X)_{n}$.

Lemma 9. The definition of $\psi$ in (7) may be rewritten as

$$
\begin{aligned}
& \psi_{n}(\tau x \cdot g)=\xi_{x_{(0,1)}} \otimes\left(\psi_{n}(g)-\sum_{i=1}^{n} s^{-1} x_{(0, \ldots, i+1)} \otimes \psi_{n-i} \omega_{i}(x, g)\right), \\
& \psi_{n}\left(\tau x^{-1} \cdot h\right)=\left(1+s^{-1} x_{(0,1)}\right) \otimes \psi_{n}(h)+\sum_{i=1}^{n} s^{-1} x_{(0, \ldots, i+1)} \otimes \psi_{n-i} \bar{\omega}_{i}(x, h),
\end{aligned}
$$

where

$$
\begin{aligned}
& \omega_{i}(x, g)=\tau d_{1}^{i} x \cdot d_{0}^{i} g \in(G X)_{n-i}, \\
& \bar{\omega}_{i}(x, h)=\omega_{i}\left(x, \tau x^{-1} \cdot h\right)=\tau d_{0} d_{2}^{i-1} x \cdot d_{0}^{i} h .
\end{aligned}
$$

Proof. Collecting the terms in (7) involving $\psi_{n}(\tau x \cdot g)$ and dividing by $1+s^{-1} x_{(0,1)}$ gives the first equation. The second is obtained by taking $g=\tau x^{-1} \cdot h$ in the first.

From these formulae it is straightforward to give the map $\psi$ explicitly in low degrees.

Lemma 10. The map $\psi_{0}:(G C X)_{0} \rightarrow(\hat{\Omega} C X)_{0}$ agrees with that defined in Proposition 4 , and the map $\psi_{1}:(G C X)_{1} \rightarrow(\hat{\Omega} C X)_{1}$ is given for $x, x_{i} \in X_{2}$ and $\alpha, \alpha_{i}= \pm 1$ by

$$
\begin{aligned}
\psi_{1}\left(\tau x_{1}^{\alpha_{1}} \ldots \tau x_{r}^{\alpha_{r}}\right) & =\sum_{i=1}^{r} \psi_{0} d_{1}\left(\tau x_{1}^{\alpha_{1}} \ldots \tau x_{i-1}^{\alpha_{i-1}}\right) \otimes \psi_{1}\left(\tau x_{i}^{\alpha_{i}}\right) \otimes \psi_{0} d_{0}\left(\tau x_{i+1}^{\alpha_{i+1}} \ldots \tau x_{r}^{\alpha_{r}}\right) \\
\text { with } \psi_{1}\left(\tau x^{\alpha}\right) & =\left\{\begin{array}{cl}
-\psi_{0}\left(\tau x_{(0,1)}\right) \otimes s^{-1} x \otimes \psi_{0}\left(\tau x_{(0,2)}\right) & (\alpha=+1) \\
s^{-1} x \otimes \psi_{0}\left(\tau x_{(1,2)}\right) & (\alpha=-1) .
\end{array}\right.
\end{aligned}
$$

Lemma 11. $\psi$ is well-defined.

Proof. We show that $\psi_{n}(w)=0$ if $w$ is degenerate, by induction on $n$ and on the word length in $G X$. Suppose $0 \leq j \leq n-1$ and

$$
w=s_{j}\left(\tau x^{\alpha} \cdot g\right)
$$


where $\alpha= \pm 1$ and $\tau x^{\alpha} \cdot g$ is a reduced word in $(G X)_{n-1}$. For $\alpha=+1$ we have

$$
\begin{aligned}
\left(1+s^{-1} x_{(0,1)}\right) & \otimes \psi_{n}(w)=\left(1+s^{-1} x_{(0,1)}\right) \otimes \psi_{n}\left(\tau s_{j+1} x \cdot s_{j} g\right) \\
& =\psi_{n} s_{j} g-\sum_{i=1}^{n}\left(s_{j+1} x\right)_{(0, \ldots, i+1)} \otimes \psi_{n-i} \omega_{i}\left(s_{j+1} x, s_{j} g\right)
\end{aligned}
$$

in which the first term is zero inductively. Each term in the summation is also zero since $\left(s_{j+1} x\right)_{(0, \ldots, i+1)}$ is degenerate for $j<i$ and $\omega_{i}\left(s_{j+1} x, s_{j} g\right)=s_{j-i} \omega_{i}(x, g)$ for $j \geq i$. Since $1+s^{-1} x_{(0,1)}$ is invertible, we have $\psi_{n}(w)=0$.

The argument for $\alpha=-1$ is similar.

2.2. Properties of the retraction map. We now prove that $\psi$ is a morphism of differential graded algebras and a retraction of the Szczarba map $\phi$.

Proposition 12. $\psi$ is a chain map.

Proof. We will show that for all $x \in X_{n+1}$ and $g \in(G X)_{n}$,

$$
\partial_{n}^{\Omega} \psi_{n}(\tau x \cdot g)=\psi_{n-1} \partial_{n}(\tau x \cdot g),
$$

by induction on $n$ and on the word length in $G X$. We first observe that

$$
\begin{aligned}
& \psi_{n-1}\left(d_{0}(\tau x \cdot g)-\tau d_{1} x \cdot d_{0} g\right)=\psi_{n-1}\left(\tau d_{0} x^{-1} \cdot \tau d_{1} x \cdot d_{0} g\right)-\psi_{n-1}\left(\tau d_{1} x \cdot d_{0} g\right) \\
& =s^{-1} x_{(1,2)} \otimes \psi_{n-1}\left(\tau d_{1} x \cdot d_{0} g\right)+\sum_{i=1}^{n-1} s^{-1} x_{(1, \ldots, i+2)} \otimes \psi_{n-1-i} \bar{\omega}_{i}\left(d_{0} x, \tau d_{1} x \cdot d_{0} g\right),
\end{aligned}
$$

using the second formula in Lemma 9 Now since $\tau d_{1} x \cdot d_{0} g=\omega_{1}(x, g)$, and also $\bar{\omega}_{i}\left(d_{0} x, \tau d_{1} x \cdot d_{0} g\right)=\bar{\omega}_{i}\left(d_{1} x, \tau d_{1} x \cdot d_{0} g\right)=\omega_{i}\left(d_{1} x, d_{0} g\right)=\omega_{i+1}(x, g)$, we get

$$
\psi_{n-1}\left(d_{0}(\tau x \cdot g)-\tau d_{1} x \cdot d_{0} g\right)=\sum_{k=1}^{n} s^{-1} x_{(1, \ldots, k+1)} \otimes \psi_{n-k} \omega_{k}(x, g)
$$

and substituting $d_{1}^{r-1} x$ and $d_{0}^{r-1} g$ for $x$ and $g$ respectively, we obtain

$$
\psi_{n-r}\left(d_{0} \omega_{r-1}(x, g)-\omega_{r}(x, g)\right)=\sum_{k=r}^{n} s^{-1} x_{(r, \ldots, k+1)} \otimes \psi_{n-k} \omega_{k}(x, g) .
$$

Now, using Lemma 9, we know that

$$
\begin{aligned}
(1+ & \left.s^{-1} x_{(0,1)}\right) \otimes \partial_{n}^{\Omega} \psi_{n}(\tau x \cdot g)=\partial_{n}^{\Omega}\left(\left(1+s^{-1} x_{(0,1)}\right) \otimes \psi_{n}(\tau x \cdot g)\right) \\
& =\partial_{n}^{\Omega}\left(-\sum_{i=1}^{n} s^{-1} x_{(0, \ldots, i+1)} \otimes \psi_{n-i} \omega_{i}(x, g)+\psi_{n} g\right) \\
& =-\sum_{i=1}^{n} \partial_{i}^{\Omega} s^{-1} x_{(0, \ldots, i+1)} \otimes \psi_{n-i} \omega_{i}(x, g) \\
& -\sum_{i=1}^{n-1}(-1)^{i} s^{-1} x_{(0, \ldots, i+1)} \otimes \psi_{n-1-i} \partial_{n-i} \omega_{i}(x, g)+\psi_{n-1} \partial_{n} g
\end{aligned}
$$


since inductively $\partial_{n-i}^{\Omega} \psi_{n-i}=\psi_{n-i-1} \partial_{n-i}$ and $\partial_{n}^{\Omega} \psi_{n} g=\psi_{n-1} \partial_{n} g$. Now expanding the operators $\partial^{\Omega}$ and $\partial$ we get

$$
\begin{aligned}
( & \left.+s^{-1} x_{(0,1)}\right) \otimes \partial_{n}^{\Omega} \psi_{n}(\tau x \cdot g) \\
= & -\sum_{i=1}^{n} \sum_{r=0}^{i+1}(-1)^{r+1} s^{-1} x_{(0, \ldots, \hat{r}, \ldots i+1)} \otimes \psi_{n-i} \omega_{i}(x, g) \\
& -\sum_{i=1}^{n} \sum_{q=1}^{i}(-1)^{q} s^{-1} x_{(0, \ldots, q)} \otimes s^{-1} x_{(q, \ldots, i+1)} \otimes \psi_{n-i} \omega_{i}(x, g) \\
& -\sum_{j=1}^{n-1} \sum_{t=0}^{n-j}(-1)^{j+t} s^{-1} x_{(0, \ldots, j+1)} \otimes \psi_{n-1-j} d_{t} \omega_{j}(x, g)+\sum_{k=0}^{n}(-1)^{k} \psi_{n-1} d_{k} g .
\end{aligned}
$$

Collecting together the terms for which either $i=1, k=0, r=0$, or $q=1$ gives

$$
\begin{aligned}
& \left(\left(1+s^{-1} x_{(0,1)}\right)-\left(1+s^{-1} x_{(0,2)}\right)\right) \otimes \psi_{n-1} \omega_{1}(x, g)+\psi_{n-1} d_{0} g \\
& +\sum_{i=1}^{n}\left(1+s^{-1} x_{(0,1)}\right) \otimes s^{-1} x_{(1, \ldots i+1)} \otimes \psi_{n-i} \omega_{i}(x, g) \\
& =\left(1+s^{-1} x_{(0,1)}\right) \otimes \psi_{n-1} d_{0}(\tau x \cdot g)-\left(1+s^{-1} x_{(0,2)}\right) \otimes \psi_{n-1} \omega_{1}(x, g)+\psi_{n-1} d_{0} g
\end{aligned}
$$

by (8), and by Lemma 9 the last two terms here cancel exactly with the terms for $r=1$ and $i>1$ in (10).

Now collecting the terms for $r=i+1$ and $i>1$ in (10), together with all the $(i, q)$-indexed terms not already considered, gives

$$
\begin{aligned}
& -\sum_{i=2}^{n}\left((-1)^{i} s^{-1} x_{(0, \ldots, i)}+\sum_{q=2}^{i}(-1)^{q} s^{-1} x_{(0, \ldots, q)} \otimes s^{-1} x_{(q, \ldots, i+1)}\right) \otimes \psi_{n-i} \omega_{i}(x, g) \\
& =-\sum_{r=2}^{n}(-1)^{r} s^{-1} x_{(0, \ldots, r)} \otimes\left(\psi_{n-r} \omega_{r}(x, g)+\sum_{k=r}^{n} s^{-1} x_{(r, \ldots, i+1)} \otimes \psi_{n-i} \omega_{i}(x, g)\right) \\
& =-\sum_{r=2}^{n}(-1)^{r} s^{-1} x_{(0, \ldots, r)} \otimes \psi_{n-r} d_{0} \omega_{r-1}(x, g)
\end{aligned}
$$

by (9), and this cancels exactly with the terms for $t=0$ in (10).

Thus expression (10) is equal to

$$
\begin{aligned}
& \left(1+s^{-1} x_{(0,1)}\right) \otimes \psi_{n-1} d_{0}(\tau x \cdot g)+\sum_{k=1}^{n}(-1)^{k} \psi_{n-1} d_{k} g \\
& -\sum_{2 \leq r \leq i \leq n}(-1)^{r+1} s^{-1} x_{(0, \ldots, \hat{r}, \ldots i+1)} \otimes \psi_{n-i} \omega_{i}(x, g) \\
& -\sum_{1 \leq j<j+t \leq n}(-1)^{j+t} s^{-1} x_{(0, \ldots, j+1)} \otimes \psi_{n-1-j} d_{t} \omega_{j}(x, g) .
\end{aligned}
$$


Now to complete the proof it remains to show that expression (11) is equal to

$$
\begin{gathered}
\left(1+s^{-1} x_{(0,1)}\right) \otimes \psi_{n-1} \partial_{n}(\tau x \cdot g)=\left(1+s^{-1} x_{(0,1)}\right) \otimes \psi_{n-1} d_{0}(\tau x \cdot g) \\
+\sum_{i=1}^{n}(-1)^{i}\left(1+s^{-1} x_{(0,1)}\right) \otimes \psi_{n-1}\left(\tau d_{i+1} x \cdot d_{i} g\right) .
\end{gathered}
$$

The first term is as required, and by Lemma 9 the summation is

$$
\sum_{i=1}^{n}(-1)^{i}\left(\psi_{n-1} d_{i} g-\sum_{k=1}^{n-1} s^{-1}\left(d_{i+1} x\right)_{(0, \ldots, k+1)} \otimes \psi_{n-1-k} \omega_{k}\left(d_{i+1} x, d_{i} g\right)\right) .
$$

The result therefore follows from the observations that

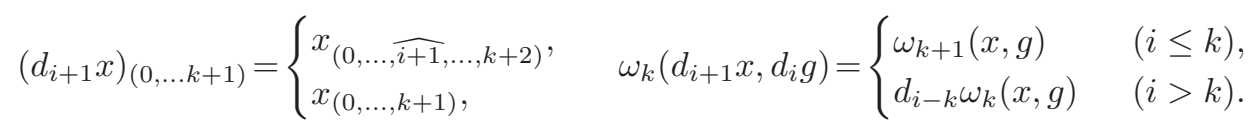

Proposition 13. The map $\psi$ is an algebra homomorphism.

Proof. Let $v \in(G X)_{p}$ and $w \in(G X)_{q}$ and consider $v \otimes w \in(C G X \otimes C G X)_{n}$, $n=p+q$. To show that $\psi$ is multiplicative we must prove that

$$
\psi_{n} m(v \otimes w)=\sum_{(\mu, \nu)}(-1)^{\operatorname{sgn}(\mu, \nu)} \psi_{n}\left(s_{\mu} v \cdot s_{\nu} w\right)=\psi_{p} v \otimes \psi_{q} w
$$

in $\hat{\Omega} C X$ by induction on $p$ and the word length of $v$. For $v=*$, or $p$ or $q=0$, there is nothing to prove; suppose inductively that $p, q \geq 1$ and $v=\tau x \cdot g$ for $x \in X_{p+1}$ (the argument for $v=\tau x^{-1} \cdot g$ is similar). Then by Lemma 9 ,

$$
\begin{aligned}
(1+ & \left.s^{-1} x_{(0,1)}\right) \otimes \psi_{n}\left(s_{\mu}(\tau x \cdot g) \cdot s_{\nu} w\right)=\left(1+s^{-1} x_{(0,1)}\right) \otimes \psi_{n}\left(\tau s_{\mu}^{\prime} x \cdot\left(s_{\mu} g \cdot s_{\nu} w\right)\right) \\
& =\psi_{n}\left(s_{\mu} g \cdot s_{\nu} w\right)-\sum_{i=1}^{n} d_{i+2}^{n-i} s_{\mu}^{\prime} x \otimes \psi_{n-i} \omega_{i}\left(s_{\mu}^{\prime} x, s_{\mu} g \cdot s_{\nu} w\right) .
\end{aligned}
$$

The term $d_{i+2}^{n-i} s_{\mu}^{\prime} x$ will be degenerate unless $i \leq p$ and $\left(s_{\mu}, s_{\nu}\right)$ is of the form $\left(s_{i+\xi_{q}} s_{i+\xi_{q-1}} \ldots s_{i+\xi_{1}}, s_{0}^{i} s_{\zeta}\right)$ for some $(p-i, q)$-shuffle $(\xi, \zeta)$, and we have

$$
\begin{aligned}
& \left(1+s^{-1} x_{(0,1)}\right) \otimes \psi_{n} m(v \otimes w) \\
& =\sum_{(\mu, \nu)}(-1)^{\operatorname{sgn}(\mu, \nu)} \psi_{n}\left(s_{\mu} g \cdot s_{\nu} w\right)-\sum_{\substack{1 \leq i \leq p \\
(\xi, \zeta)}}(-1)^{\operatorname{sgn}(\xi, \zeta)} d_{i+2}^{p-i} x \otimes \psi_{n-i}\left(s_{\xi} \omega_{i}(x, g) \cdot s_{\zeta} w\right) \\
& =\psi_{n} m(g \otimes w)-\sum_{i=1}^{p} d_{i+2}^{p-i} x \otimes \psi_{n-i} m\left(\omega_{i}(x, g) \otimes w\right) \\
& =\left(\psi_{p} g-\sum_{i=1}^{p} d_{i+2}^{p-i} x \otimes \psi_{p-i} \omega_{i}(x, g)\right) \otimes \psi_{q} w, \quad \text { by the inductive hypothesis, } \\
& =\left(1+s^{-1} x_{(0,1)}\right) \otimes \psi_{p} v \otimes \psi_{q} w,
\end{aligned}
$$

by Lemma 9 The result follows.

Proposition 14. The map $\psi$ is a retraction of $\phi$; that is, the composite $\psi \phi$ is the identity. 
Proof. It is enough to prove this on algebra generators of $\hat{\Omega} C X$. For $x \in X_{n+1}$ and $i=\left(i_{1}, \ldots, i_{n}\right) \in S_{n}$ we will show that

$$
\psi_{n} \mathrm{Sz}_{i} x= \begin{cases}x & \text { if } i_{1}=\cdots=i_{n}=0 \\ 0 & \text { otherwise. }\end{cases}
$$

Denote by $x_{0 ; i}$ the element of $X$ satisfying

$$
D_{0 ; i}^{n+1} \tau x=\tau x_{0 ; i}
$$

Lemma 8 tells us that $\psi_{n}\left(\tau x_{0 ; i} \cdot \mathrm{Sz}_{i} x\right)=0$, and so by Lemma 9 we have

$$
\psi_{n} \mathrm{Sz}_{i} x=\sum_{k=1}^{n} d_{k+2}^{n-k} x_{0 ; i} \otimes \psi_{n-k} \omega_{k}\left(x_{0 ; i}, \mathrm{Sz}_{i} x\right) .
$$

From (6) we see that $D_{0, i}^{n+1} \tau x$ has an $s_{k-1}$ degeneracy if $i_{k} \neq 0$. Thus $d_{k+2}^{n-k} x_{0 ; i}$ is degenerate except in the case $i_{1}=\cdots=i_{k}=0$. In this case we see from (6) and Lemma 6 that

$$
\omega_{k}\left(x_{0 ; i}, \mathrm{Sz}_{i} x\right)=\tau d_{1}^{k} x_{0 ; i} \cdot d_{0}^{i} \mathrm{Sz}_{i} x=D_{0 ; i_{k+1}, \ldots, i_{n}}^{n-k+1} \tau d_{1}^{k} x \cdot \mathrm{Sz}_{i_{k+1}, \ldots, i_{n}} d_{1}^{k} x,
$$

which is degenerate again by Lemma 8 . The only nonzero term is therefore

$$
\psi_{n} \mathrm{Sz}_{0, \ldots, 0} x=x_{0 ; 0, \ldots, 0} \otimes \psi_{0}(*)=x,
$$

and hence $\psi \phi x=x$, as required.

\section{Deformation Retraction of the loop Group}

Both the Kan functor $G$ and the cobar construction model loop spaces. In the 1-reduced case it is easy to show that the Szczarba map $\phi: \Omega C X \rightarrow C G X$ is a weak equivalence, by applying Zeeman's comparison theorem to the map of spectral sequences associated with

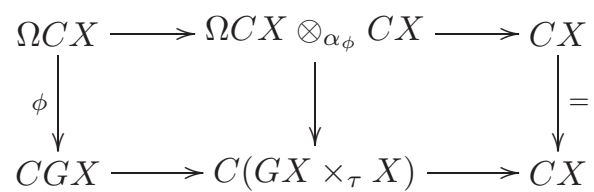

in which the total spaces are acyclic.

We prove here the following stronger result.

Theorem 15. Let $X$ be a 0-reduced simplicial set. Let $\phi$ be the Szczarba map and $\psi$ the retraction map defined above.

There is a natural strong deformation retraction of chain complexes

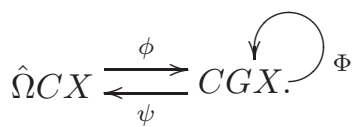

Recall that if $A$ and $B$ are chain complexes, $\nabla: A \rightarrow B$ and $f: B \rightarrow A$ are chain maps, and $h: B \rightarrow B$ is linear map of degree +1 , then

$$
A \underset{f}{\stackrel{\nabla}{\rightleftarrows}} \stackrel{\bigcap_{h}}{\longrightarrow}
$$


is a strong deformation retract if $f \nabla=I d_{A}$ and $\partial h+h \partial=\nabla f-I d_{B}$. Given a strong deformation retract, one can apply the machinery of homological perturbation theory to transfer perturbations of the structure $B$ across to $A$, obtaining a new strong deformation retract; see [4], [5], 8].

Proof. According to Proposition 14, we need only to prove that there is a natural chain homotopy from the composite $\phi \psi$ to the identity map on $C G X$. The proof, an acyclic models argument, proceeds by induction on the degree.

The base step of the induction is trivial, by Proposition 4 . We can simply set $\Phi_{0}=0: C_{0} G X \rightarrow C_{1} G X$ for all 0-reduced simplicial sets $X$.

Suppose now that $\Phi_{k}: C_{k} G X \rightarrow C_{k+1} G X$ has been defined for all $0 \leq k<n$ and for all 0-reduced simplicial sets $X$ so that

(1.k) $\Phi_{k}$ is natural in $X$ for all $k$, and

(2.k) $\partial \Phi_{k}+\Phi_{k-1} \partial=\phi \psi-I d_{C G X}$ for all $k$ and all $X$,

where $n \geq 1$.

Let $\bar{\Delta}[n]$ denote the quotient of the standard simplicial $n$-simplex by its 0 skeleton. If $x=\left(k_{0} \cdots k_{j}\right)$ is a $j$-simplex of $\Delta[n]$, let $x \cdot n$ denote the $(j+1)$-simplex $\left(k_{0} \cdots k_{j} n\right)$. Let

$$
h_{i}^{n}:(G \bar{\Delta}[n])_{i} \rightarrow(G \bar{\Delta}[n])_{i+1}
$$

denote the group homomorphism specified by $h_{i}^{n}(\tau x)=\tau(x \cdot n)$ for all $x \in \bar{\Delta}[n]_{i+1}$. Let

$$
\bar{h}^{n}: C_{*} G \bar{\Delta}[n] \rightarrow C_{*+1} G \bar{\Delta}[n]
$$

denote the degree +1 linear map specified by $\bar{h}_{i}^{n}(w)=-h_{i}^{n}(w)$ for all $w \in(G \bar{\Delta}[n])_{i}$.

Morace and Prouté proved in [10] that for all $i \geq 1$,

$$
\partial_{i+1} \bar{h}_{i}^{n}+\bar{h}_{i-1}^{n} \partial_{i}=I d,
$$

i.e., that $\bar{h}^{n}$ is a contraction in positive degrees. It follows that $H_{i} G \bar{\Delta}[n]=0$ for all $i \geq 1$.

Consider the infinite wedge

$$
W(n+1)=\bigvee_{m \in \mathbb{N}} \bar{\Delta}[n+1]
$$

There is a chain homotopy

$$
\tilde{h}^{n+1}: C_{*} G W(n+1) \rightarrow C_{*+1} G W(n+1)
$$

that is a contraction in positive degrees and that generalises Morace and Prouté's construction.

Let

$$
w=\tau \delta_{m_{1}}^{\alpha_{1}} \cdots \tau \delta_{m_{k}}^{\alpha_{k}} \in(G W(n+1))_{n}
$$

where $\delta_{m_{i}}$ denotes the unique nondegenerate $(n+1)$-simplex in the $m_{i}^{\text {th }}$ copy of $\bar{\Delta}[n+1]$ in $W(n)$, and $\alpha_{i}= \pm 1$. Set

$$
\Phi_{n}(w)=\tilde{h}^{n}\left(\phi \psi(w)-w-\Phi_{n-1}(\partial w)\right) .
$$

The induction hypothesis implies that $\phi \psi(w)-w-\Phi_{n-1}(\partial w)$ is a cycle and that

$$
\begin{aligned}
\partial \Phi_{n}(w) & =-\tilde{h}^{n} \partial\left(\phi \psi(w)-w-\Phi_{n-1}(\partial w)\right)+\phi \psi(w)-w-\Phi_{n-1}(\partial w) \\
& =\phi \psi(w)-w-\Phi_{n-1}(\partial w) .
\end{aligned}
$$


Adding $\Phi_{n-1}(\partial w)$ to both sides of this equation, we obtain

$$
\partial \Phi_{n}(w)+\Phi_{n-1}(\partial w)=\phi \psi(w)-w ;
$$

i.e., (2.n) holds for all such $w$.

Let $X$ be any 0-reduced simplicial set, and let

$$
w=\tau x_{1}^{\alpha_{1}} \cdots \tau x_{k}^{\alpha_{k}}
$$

be any nondegenerate $n$-simplex in $G X$, where $\alpha_{i}= \pm 1$ for all $i$. Let $\zeta_{i}: \bar{\Delta}[n+1] \rightarrow$ $X$ be the simplicial map representing $x_{i}$.

Let $\gamma: \bar{\Delta}[n+1] \rightarrow X$ denote the simplicial map collapsing everything to the basepoint. Consider the morphism of simplicial groups

$$
\Psi_{w}=G\left(\zeta_{1} \vee \cdots \vee \zeta_{k} \vee \bigvee_{m>k} \gamma\right): G W(n+1) \rightarrow G X .
$$

Observe that

$$
\Psi_{w}\left(\tau \delta_{1}^{\alpha_{1}} \cdots \tau \delta_{k}^{\alpha_{k}}\right)=w,
$$

where $\delta_{i}$ denotes the unique nondegenerate $n$-simplex in the $i^{\text {th }}$ copy of $\bar{\Delta}[n+1]$ in $W(n+1)$.

Using the map $\Psi_{w}$ constructed above for any generator $w$ of $C_{n} G X$, we define $\Phi_{n}: C_{n} G X \rightarrow C_{n+1} G X$ for any 0 -reduced simplicial set $X$ by

$$
\Phi_{n}(w)=C_{n+1} \Psi_{w} \circ \Phi_{n}\left(\tau \delta_{1}^{\alpha_{1}} \cdots \tau \delta_{k}^{\alpha_{k}}\right) .
$$

Note that if $X=W(n+1)$ and $w=\tau \delta_{j_{1}}^{\alpha_{1}} \cdots \tau \delta_{j_{k}}^{\alpha_{k}}$, then

$$
\Psi_{w}: G W(n+1) \rightarrow G W(n+1)
$$

is a homomorphism of simplicial groups given simply by permuting generators. It follows from the construction of the chain homotopy $\bar{h}^{n+1}$ and therefore of the chain homotopy $\tilde{h}^{n+1}$ that $\tilde{h}^{n+1}$ is natural with respect to homomorphisms that simply permute generators, so that

$$
C_{n+1} \Psi_{w} \circ \tilde{h}_{n}^{n}=\tilde{h}_{n}^{n} \circ C_{n} \Psi_{w} .
$$

Consequently, $\Phi_{n}(w)$ is indeed well-defined, since $\phi, \psi$ and, by the induction hypothesis, $\Phi_{n-1}$ are all natural with respect to simplicial maps.

Moreover,

$$
\begin{aligned}
\partial \Phi_{n}(w) & =C_{n+1} \Psi_{w} \circ \partial \Phi_{n}\left(\tau \delta_{1}^{\alpha_{1}} \cdots \tau \delta_{k}^{\alpha_{k}}\right) \\
& =C_{n+1} \Psi_{w} \circ\left(\left(\phi \psi-I d_{C G W(n)}-\Phi_{n-1} \partial\right)\left(\tau \delta_{1}^{\alpha_{1}} \cdots \tau \delta_{k}^{\alpha_{k}}\right)\right) \\
& \stackrel{(\star)}{=}\left(\phi \psi-I d_{C G X}-\Phi_{n-1} \partial\right) \circ C_{n} \Psi_{w}\left(\tau \delta_{1}^{\alpha_{1}} \cdots \tau \delta_{k}^{\alpha_{k}}\right) \\
& =\left(\phi \psi-I d_{C G X}-\Phi_{n-1} \partial\right)(w),
\end{aligned}
$$

where the equality $(\star)$ follows from naturality of $\phi, \psi$ and $\Phi_{n-1}$. In other words,

$$
\partial \Phi_{n}+\Phi_{n-1} \partial=\phi \psi-I d_{C G X},
$$

for all $X$; i.e., condition (2.n) holds.

To conclude, observe that condition (1.n) holds as well, since for all simplicial maps $g: X \rightarrow Y$ between 0-reduced spaces and all $w \in G X$,

$$
G g \circ \Psi_{w}=\Psi_{G g(w)}: G W(n+1) \rightarrow G Y .
$$


Remark 16. It is in order to be able to apply the chain homotopy of Morace and Prouté that we work with 0-reduced simplicial sets. There is no such chain homotopy in the 1-reduced case, so it seems we are obliged to prove the existence of the strong deformation retract in the 0 -reduced case in order to conclude that it exists in the 1-reduced case as well.

Remark 17 . As defined in the proof above, $\Phi_{n}$ is almost certainly not a derivation homotopy, since, as easy computations show, $\tilde{h}$ is not a derivation homotopy.

Remark 18. Morace and Prouté showed that $\bar{h}_{i+1}^{n} \circ \bar{h}_{i}^{n}=0$ for all $i$ and $n$, from which it follows that $\tilde{h}_{i+1}^{n} \circ \tilde{h}_{i}^{n}=0$ as well and therefore that

$$
\tilde{h}_{n+1}^{n+1} \circ \Phi_{n}\left(\tau \delta_{j_{1}}^{\alpha_{1}} \cdots \tau \delta_{j_{k}}^{\alpha_{k}}\right)=0
$$

for all $j_{1}, \ldots, j_{k}$ and $\alpha_{1}, \ldots, \alpha_{k}$.

Remark 19. The results in this paper generalise from chain complexes to crossed complexes. There is a crossed cobar construction $\Omega \pi X$ on the fundamental crossed complex $\pi X$, see [2], and we may define a 'crossed' Szczarba map of crossed chain algebras $\phi: \Omega \pi X \rightarrow \pi G X$ that forms part of a deformation retraction

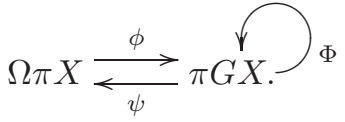

The classical argument that $\phi$ is a weak equivalence, using (12), does not go through in this slightly nonabelian situation, since it seems there is no good notion of twisted tensor product of crossed complexes.

\section{ACKNOWLEDGEMENT}

The second author would like to warmly thank the Centre de Recerca Matemàtica University for the excellent working conditions provided in the final stages of this paper. He is partially supported by MEC/FEDER grant MTM2007-63277.

\section{REFERENCES}

1. J. F. Adams, On the cobar construction, Proc. Nat. Acad. Sci. U.S.A. 42 (1956), 409-412. MR.0079266 (18:59c)

2. Hans-Joachim Baues and Andrew Tonks, On the twisted cobar construction, Math. Proc. Cambridge Philos. Soc. 121 (1997), no. 2, 229-245. MR1426521(97k:55023)

3. V. K. A. M. Gugenheim and H. J. Munkholm, On the extended functoriality of Tor and Cotor, J. Pure Appl. Algebra 4 (1974), 9-29. MR0347946 (50:445)

4. V.K.A.M. Gugenheim, L. Lambe, and J. Stasheff, Perturbation theory in differential homological algebra, Illinois J. Math. 33 (1989), 566-582. MR1007895(91e:55023)

5. _ Perturbation theory in differential homological algebra. II, Illinois J. Math. 35 (1991), 357-373. MR 1103672 (93e:55018)

6. Kathryn Hess, Paul-Eugène Parent, and Jonathan Scott, A chain coalgebra model for the James map, Homology, Homotopy Appl. 9 (2007), no. 2, 209-231. MR.2366950(2008k:55020)

7. Daniel M. Kan, Abstract homotopy. IV, Proc. Nat. Acad. Sci. U.S.A. 42 (1956), 542-544. MR0087938 (19:440b)

8. L. Lambe and J. Stasheff, Applications of perturbation theory to iterated fibrations, Manuscr. Math. 58 (1987), 363-376. MR893160 (89d:55041)

9. J. Peter May, Simplicial objects in algebraic topology, Chicago Lectures in Mathematics, University of Chicago Press, Chicago, IL, 1992, reprint of the 1967 original. MR 1206474 (93m:55025) 
10. Frédéric Morace and Alain Prouté, Brown's natural twisting cochain and the Eilenberg-Mac Lane transformation, J. Pure Appl. Algebra 97 (1994), no. 1, 81-89. MR.1310750(96a:55029)

11. R. H. Szczarba, The homology of twisted cartesian products, Trans. Amer. Math. Soc. 100 (1961), 197-216. MR0137111 (25:567)

Institut de GÉométrie, algèbre et topologie (IGAT), École Polytechnique Fédérale De Lausanne, CH-1015 Lausanne, Switzerland

E-mail address: kathryn.hess@epfl.ch

Statistics, OR and Mathematics Research Centre (StORM), London Metropolitan University, 166-220 Holloway RoAd, London N7 8DB, United Kingdom

E-mail address: a.tonks@londonmet.ac.uk 